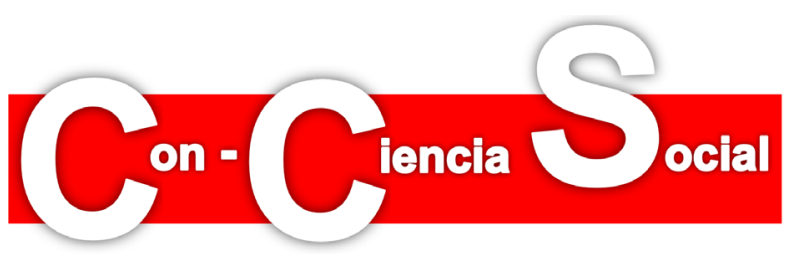

\title{
El patio de los mandarines: ensayo sobre el fetichismo académico
}

\section{The courtyard of the mandarins: essay on academic fetishism}

\author{
José Carlos Bermejo Barrera \\ Universidade de Santiago de Compostela \\ josecarlos.bermejo@usc.es
}

Recibido en septiembre de 2018

Aceptado en noviembre de 2018

DOI:10.7203/con-cienciasocial.3.16788

\begin{abstract}
RESUMEN
El mundo económico está viviendo un proceso de transformación global con el desarrollo de la economía financiera global. Ese cambio afecta de un modo directo a las universidades y a los procesos de producción del conocimiento. No obstante, en el caso español esto no es así porque sus universidades son esencialmente universidades públicas controladas por funcionarios, que no están sufriendo cambios ni ajustes al mercado. En las universidades españolas se está desarrollando una ideología que ha creado el fetichismo del mercado como instrumento para conseguir el control y la alienación de las comunidades académicas. Esa ideología es una representación invertida de la realidad académica. Se lleva a cabo un estudio del panorama general de la economía global y se muestra, siguiendo los conceptos marxistas de ideología, fetichismo y alienación como se estructuran las relaciones de poder en las universidades españolas.
\end{abstract}

Palabras clave: economía global, ideología, fetichismo, alienación, universidades españolas.

\section{Referencia}

Bermejo Barrera, J. C. (2020). El patio de los mandarines: ensayo sobre el fetichismo académico. Con-Ciencia Social (segunda época), 3, 9-30. DOl:10.7203/concienciasocial.3.16788

\begin{abstract}
The economic world is currently in a global process of transformation with the growth of the financial global economy. This change is affecting universities and the production of knowledge. However, this process is very different in Spain because Spanish universities are public universities leaded by public servers independent of the market. In Spanish universities, the ideology of the fetishism of the market is growing as an apparatus of control and alienation of the academic communities. This ideology is an inverse representation of reality. This paper studies the trends of the world economy and how power relations are structured at Spanish universities, according the Marxist concepts of ideology, fetishism and alienation.
\end{abstract}

Keywords: global economy, ideology, fetishism, alienation, Spanish universities. 
Suele decirse que los historiadores son profetas que únicamente saben adivinar el pasado, lo cual no tiene mucho mérito, pues también se dice que, como el pasado ya pasó nadie puede cambiarlo ya, ni siquiera Dios. Por eso que un historiador intente predecir por qué senderos transitarán las universidades españolas en los próximos años podría parecer una osadía sin sentido. Desde hace más de diez años he venido tratando el tema de las universidades españolas en una serie de libros, y será a partir de los datos y las tesis defendidas en ellos de donde tomaré el punto departida de esta reflexión (véase Bermejo Barrera, 2008, 2009, 2011, 2012, 2015, 2017).

Debemos partir del principio de que todo conocimiento es limitado y transitorio (Bermejo Barrera, 2010). Ello se debe a que nuestros conocimientos empíricos parten de un limitado número de observaciones realizadas frente al número casi infinito de observaciones posibles. Además, esas observaciones, en las ciencias experimentales, dependen de nuestros aparatos y sistemas de información, que son muy limitados, en tanto que no pueden registrar más que fenómenos de determinados tipos, dependiendo del grado de desarrollo de nuestras tecnologías. Pero a ello tenemos que añadir también que, a su vez, lo que podemos observar depende de nuestros sistemas de conceptos lógicos y científicos, pues son ellos los que nos permiten desarrollar los sistemas de análisis y tratamiento de la información que podemos recopilar. $\mathrm{Y}$ del mismo modo esos conceptos son también muy limitados, puesto que dependen del desarrollo del pensamiento lógico matemático y del pensamiento filosófico de cada momento histórico. Decía Kant que las intuiciones sin los conceptos son ciegas, pero que los conceptos sin intuiciones están vacíos, y por eso el desarrollo de las ciencias y del conocimiento en general está condicionado por el despliegue en el tiempo de esta interrelación dialéctica, que va unida a la sistematización de nuestras ideas acerca del mundo, el hombre y la historia.

En los campos de la historia y las ciencias sociales no es posible formar conceptos de validez universal, porque se da el caso de que coinciden el sujeto y el objeto del conocimiento, y porque nuestras datos, observaciones e ideas están fuertemente vinculados con los valores morales y políticos. Por esta razón, si es verdad que en el campo de las ciencias naturales debemos siempre saber distinguir entre lo que se puede conocer: los fenómenos; y la totalidad de la realidad: el nóumeno, y ser conscientes de que nuestro conocimiento no es más que un pequeño haz de luz en un universo de tinieblas, quedando siempre una gran zona oscura a nuestro alrededor. Con más razón en el campo de las ciencias sociales y humanas deberemos ser conscientes de que hay numerosas zonas oscuras que nos rodean, y 
también en el interior de nosotros mismos, porque al conocimiento por sí mismo se une siempre el interés económico, político, ideológico y social, que pueden hacer que, además de no ser plenamente capaces de conocernos a nosotros mismos, queramos engañarnos deliberadamente, creando una imagen propia que oculte nuestra verdadera identidad.

Fue Karl Marx, siguiendo la filosofía de Ludwig Feuerbach, quién planteó este problema para los campos de la economía y la historia en su teoría del fetichismo de la mercancía, que está muy unida a sus teorías de la ideología y la alienación, dos conceptos que serán claves en nuestro análisis de la universidad española actual. En el libro primero de su principal obra Marx (2017, pp. 83-204) desarrolló su teoría del fetichismo de la mercancía, partiendo de la noción de fetiche que había creado Charles Des Brosses en su obra Du Culte des Dieux fetiches, publicada en 1760. Tomando la palabra portuguesa feitiço, hechizo, con la que los viajeros portugueses en su camino a la India habían pasado a designar a las estatuas y objetos de culto de la zona de Guinea, este autor llegó a la conclusión de que los seres humanos tendemos a reificar nuestras ideas en objetos externos, pasando a creer que lo que no son más que nuestras opiniones, miedos o gustos son realidades inmutables inapelables. $Y$ eso mismo ocurriría, según Marx, con el capitalismo en el caso de la noción de mercancía. Para Marx en la formación del precio de una mercancía intervendrían los siguientes parámetros: capital fijo, capital circulante, coste de la fuerza de trabajo y plusvalía. Aquello que hace que un objeto se convierta en mercancía es que pase de tener un mero valor de uso a tener un valor de cambio, y ese valor depende de la única fuente capaz de crearlo que es el trabajo humano. Por esa razón la plusvalía o plus-valor no sería más que la parte del valor trabajo que el propietario de los medios de producción detrae del trabajo físico e intelectual de sus trabajadores. Marx fue consciente al final de su vida de que la plusvalía como magnitud económica no se podía cuantificar con los instrumentos matemáticos de los que él disponía. Y por esa razón dedicó la última parte de su vida al estudio del cálculo diferencial, cifrando en ello su esperanza de convertir una idea moral en un concepto económico. Su prematura muerte le impidió ver que esto habría sido un esfuerzo inútil, pero ello no quita valor a su obra como estudio de historia y de sociología. El cálculo de la plusvalía es imposible porque el cálculo económico es muy limitado en general, y porque este concepto es discutible.

La economía global de la humanidad es la sucesión de procesos termodinámicos de interrelación entre cada sociedad humana y su medio ecológico, como ha señalado Nicholas Georgescu-Roetgen (1971), y por esa razón en los procesos productivos solo 
se puede computar aquellos elementos que forman parte de los flujos de gastos e ingresos, quedando la mayor parte de los factores de producción que proporciona la naturaleza al margen del cálculo, como ha mostrado de modo nítido José Manuel Naredo (2003). Y es que está muy claro que existe una explotación en el trabajo asalariado y de otros tipos, como por ejemplo en el esclavismo y el sistema feudal, aunque no se pueda cuantificar. Si se niega esta realidad moral y jurídica es porque existe la ideología, que consiste en una representación deformada o invertida de las relaciones sociales reales, con el fin de justificar las desigualdades de todo tipo. Marx y Friedrich Engels (2014) intentaron sistematizar este concepto de ideología como mundo al revés en el libro que abandonaron "a la crítica de los ratones", una vez escrito entre 1845-1856: La ideología alemana ${ }^{1}$. Los conceptos de ideología y alienación están íntimamente unidos. La ideología es un sistema elaborado de pensamiento que, como el delirio del paranoico, crea un mundo paralelo a la realidad, pero al contrario que ese delirio, que es individual, la ideología es compartida por un amplio grupo social, o por toda una sociedad. La ideología crea un mundo paralelo en la imaginación colectiva, pero también hace que cada persona se vuelva incapaz de percibirse a sí misma dentro del mundo social, confundiéndose con la imagen falsa que la sociedad a la pertenece quieren que se haga de sí misma. Esa imagen es falsa, pero además condiciona la conducta de cada cual, haciendo que ajuste su vida a la representación que hace de ella, y limitando su libertad, como los hombres de Guinea de Des Brosses, que creaban sus fetiches para luego creer que eran superiores a ellos mismos. Los hombres han creado a los dioses, decían Demócrito y Leucipo, dos filósofos a los que Marx había dedicado su tesis doctoral, pero esos dioses acaban por dominar a los hombres infundiéndoles el temor en sus vidas y en sus sueños. Por eso dijo en un determinado momento San Agustín: ubi terror, ibi salus. De dónde está el temor, de ahí viene nuestra salvación. Esto es lo que ocurre con la alienación religiosa, pero también con la alienación y el fetichismo académicos de las universidades españolas.

La alienación académica comienza por desarrollar una ideología sobre el valor de la economía y el papel que a las universidades les corresponde en ellas. Veamos cómo lo hace. Deberíamos comenzar por destacar que en la historia del pensamiento

\footnotetext{
${ }^{1}$ En relación con ese concepto son muy útiles como introducción los libros de John Plamenatz (1983) y Sarah Kofman (1975). Sobre el pensamiento moral de Marx es esencial el libro de Philip J. Kain (1988); y sobre su juvenil teoría de la alienación el libro de David Leopold (2007). En este sentido también es muy útil el libro de Elizabeth Anderson (1993).
} 
José Carlos Bermejo Barrera, El patio de los mandarines...

económico la economía ha intentado constituirse como ciencia tomando como modelo el de la ciencia dominante en cada momento histórico. Adam Smith tomó como modelo la física newtoniana y su universo armónico, regular y regido por la doble fuerza gravitatoria de la atracción y la repulsión, para crear su idea del mercado autorregulado mediante la doble fuerza de la oferta y la demanda. Una fuerza analizable de acuerdo con modelos matemáticos, como los que proporcionaba el cálculo diferencial newtoniano (véase Passet, 2010 y Schumpeter, 1954). Al modelo newtoniano del mercado estable le sucedió el modelo darwinista de cariz biológico. Según él la economía forma parte de la lucha por la vida y está regida por el principio de la supervivencia del más apto. Un principio tautológico que afirma sobrevive el más apto, porque los supervivientes fueron los más aptos para sobrevivir. Ese fue el mundo en el que le tocó vivir a Marx, quien creyó sinceramente que Darwin habría descubierto la ley que rige la vida, después de que Newton lo hubiese hecho con la ley del mundo inorgánico.

La economía actual no se concibe basándose en esos principios, sino en otros muchos más complejos, que dependen de la idea de que la historia económica es en gran parte historia monetaria, siguiendo el modelo trazado por Milton Friedman y Jacobson Schwart $(1971)^{2}$. De acuerdo con ese modelo la riqueza global de un país se puede medir en una cifra monetaria que sería el Producto Interior Bruto. Ese producto se compone de los siguientes parámetros: consumo, inversión, gasto público y equilibrio entre exportación e importación. EI PIB es cuantificable en una magnitud monetaria, de tal modo que:

$M x V=P I B \times I$

Siendo M la masa monetaria, o cantidad de moneda emitida; $V$ su velocidad de circulación; e I la inflación. Es fácil comprender que el aumento de la masa monetaria debe generar inflación y que el control del precio del dinero, o tipo de interés por parte de los bancos centrales es una herramienta económica básica de la política económica de un país, puesto que la baja del precio del dinero favorece la inversión, y consiguientemente la producción y el consumo.

Si queremos comprender como funciona el capitalismo actual deberemos tener en cuenta que se basa en los siguientes principios, sistematizados en un libro ya clásico por Werner Sombart (1967 [1916]). En primer lugar, frente al principio de la mera satisfacción de las necesidades, que reguló casi todas las economías a lo largo

2 Para el desarrollo macroeconómico global, véase Angus Madison (2007). 
de la historia, el capitalismo establece el principio de adquisición en sí mismo, que lleva unida la necesidad de racionalizar la economía constantemente para incrementar el beneficio individual de la empresa o el individuo en un mercado competitivo. Esto será posible, como ya había señalado también Marx, sólo si la economía se desvincula de las restricciones sociales y trabas legales y se hace privada. La propiedad privada establece las diferencias básicas entre ricos y pobres, empresarios y trabajadores y demanda una estructura profesional abierta, que facilite la movilidad y el intercambio. Toda la economía se ha de centrar en el intercambio, en el mercado de bienes, servicios y capitales de todo tipo. Y además las empresas pasarán a ser de propiedad privada, individual o anónima. El capitalismo necesita crear mercados de algodón, de productos químicos o de bienes de consumo masivo, tal y como ocurrió con la primera revolución industrial inglesa y con el desarrollo del capitalismo global tras la Segunda Guerra Mundial (Beckert, 2015; de Gracia, 2005). Lo logra revolucionando las técnicas, creando nuevas demandas artificiales y favoreciendo la llamada destrucción creativa: la sustitución de un producto por otro, casi siempre de menor duración y calidad. El capitalismo depende la constante innovación tecnológica para conquistar y crear nuevos mercados. Y aquí es donde se quiere atribuir un papel clave a las universidades públicas en la ideología del fetichismo académico. Y es que no deja de ser curioso el contraste entre los factores básicos que hicieron posible la aparición del capitalismo y el mundo de las universidades públicas, financiadas con el presupuesto general del Estado y formadas en lo esencial por funcionarios.

Para que el capitalismo fuese posible fue necesario en primer lugar que se desarrollasen sistemas de comunicaciones y transporte a media y larga distancia, que permitiesen, por ejemplo, la llegada del algodón de los EEUU a las fábricas inglesas y la invasión del mundo por los tejidos de algodón ingleses. Pero ese sistema de comunicación tuvo que ir unido a un sistema de recopilación de la información por parte de los comerciantes y empresarios, ya desde el siglo XVI (Miller, 2015). Los flujos de mercancías, materias primas e información sólo pudieron integrarse en el sistema capitalista cuando los obstáculos sociales, jurídicos y económicos permitieron el desarrollo del comercio y la creación sin trabas de empresas. Unas empresas organizadas por el principio de la búsqueda de la ganancia individual, regida por el egoísmo racional. Un principio que nuestros ideólogos académicos transforman de un modo sui generis.

Todo lo que estamos viendo fue verdad hasta un determinado momento. El momento en el que el capitalismo productivo comenzó a ser desplazado por el 
capitalismo financiero, en el que los beneficios son muchos más rápidos y más cuantiosos, y en el que el modelo que intenta imitar la ciencia económica es la física de partículas. Este nuevo capitalismo trajo a su vez un proceso de desindustrialización que comenzó tras la crisis del petróleo de los años setenta del pasado siglo y ha hecho que un país como los EE.UU., o Inglaterra, sean cada vez más países desindustrializados y más orientados a la economía financiera y a la prestación de servicios de todo tipo. Judith Stein (2010) ha estudiado en el caso de los EE.UU. este proceso. En estos procesos el ascenso de la China a la India como productores es imparable, de tal modo que el mayor PIB del mundo en el año 2030 corresponderá a la China, según las estimaciones de Angus Madison (2007). La economía financiera actual es una economía global que abarca todo el planeta y funciona de modo ininterrumpido veinticuatro horas al día gracias a la revolución digital. En ella están integradas todos los mercados de capitales del mundo, siendo estos a su vez: mercados de activos financieros correspondientes a empresas productivas, de servicios o la compra de materias primas, de combustibles y de productos agrícolas, ya sean reales o en compra de futuros. Junto a ellos se compra de modo continuo monedas y deuda pública. El sistema monetario mundial se articula en cuatros ejes: el del euro, llamado el IV Reich irónicamente, el del dólar, la libra y el yen, el del yuan, y el del rublo, el real brasileño otros núcleos menores. Todo este sistema se articula en un mercado global de muy difícil análisis detallado, pero que se puede intentar formalizar del modo siguiente, según el modelo de Nicholas Dunbar (2011). Ya Gauss habría descubierto que la bolsa de valores funciona de acuerdo con el modelo estadístico de su famosa campana. Yo compro una acción a un valor muy bajo, espero a que alcance su valor máximo previsto y en ese momento vendo, provocando su pérdida del valor para poder comprarla otra vez. Si represento el proceso en unas coordenadas cartesianas con el precio en uno de sus ejes y el tiempo en el otro podré ver que la sucesión de campanas es uno onda, con su frecuencia y longitud propias, y podré estudiarla con los modelos de la física de partículas si tengo en cuenta que cada onda nunca vieja sola, sino con otros muchos miles de ondas más. Si cambio los parámetros que definen a las partículas: masa, carga, spin... por los parámetros que obtengo de la información de los mercados podré hacer modelos estadísticos que predigan el funcionamiento de la bolsa, e incluso programar ordenadores para que compren y vendan accionas automáticamente, cosa que ya se hace. Si coordino muchas ondas en una cópula gaussiana hago oscilar los valore de un grupo de 
acciones de tal modo que unos se equilibren con otros, y así obtendré un modelo estable del mercado bursátil.

El problema es que el dinero tiene un límite, como todo lo demás, aunque se multiplique en el tiempo. Toda la economía es crédito, es verdad, y el dinero solo vale en el futuro, cuando se gasta, pero la expansión de la masa monetaria y su velocidad de circulación tienen que tener un límite, para que no estallen las burbujas especulativas, como la primera conocida, la de los tulipanes en la Holanda del siglo XVII. Todos los préstamos tienen un vencimiento y por eso estallan también las estafas piramidales. Es un problema matemático y ecológico. El sistema económico es un organismo, y todo organismo tiene un límite de crecimiento, más allá del cual colapsa. Todas las ondas tienen una longitud y una frecuencia. Si su longitud tiende al infinito su frecuencia también lo hará, y ya no serán ondas. Un banco tiene un activo propio circulante y otro consolidado y unos depósitos que remunere o no, siempre tienen que cuadrar en el balance. $\mathrm{Y}$ es que el dinero es crédito, pero crédito para conseguir más crédito hasta un punto, y no hasta el infinito, porque en algún momento el dinero tiene que volver a servir para lo que nació, para intercambiar mercancías y equilibrar en el tiempo la producción y el consumo en el mundo físico. Y es que, como dice el refrán: "la mortaja no tiene bolsillos". O lo que es lo mismo: el dinero no puede sobrevivir más allá de su propio ciclo vital. Cuando la avaricia se apodera de la economía nace el mundo de la cleptopía (Taibi, 2011) y se muere el propio capitalismo. El mayor enemigo del capitalismo no es el comunismo, sino que es la avaricia y el descontrol del dinero al convertirse en dinero sucio. Raymond W. Barker (2005), un banquero norteamericano, lo ha explicado con claridad, y también otros autores (Levitt y Dunbar, 2005). En el mundo de la economía financiera global es mucho más rentable comprar y vender activos financieros que invertir en procesos productivos. $\mathrm{Y}$ uno de esos activos son las deudas públicas, que hacen que los bancos sean el sustento del Estado como la soga es el sustento del ahorcado. Estas deudas se deben en primer lugar al enorme incremento del gasto público que se origina por el desarrollo del estado del bienestar, que cubre, no solo los servicios tradicionales de seguridad, defensa y justicia, sino también la educación, la sanidad y los sistemas de jubilación (Lindert, 2011; Groeben, 2012). Hasta aquí no habría problema, si no fuese porque el trasvase del capital productivo al capital financiero puro favorece la desindustrialización de los países ricos y provoca la pérdida global de los salarios, al bajar la masa salarial. La bajada de los salarios trae consigo la bajada del consumo, y consecuentemente el incremento del paro y del empleo de baja calidad. Pero, como 
se da el caso de que los ingresos de los estados de los países ricos se obtienen básicamente de dos impuestos: el IRPF y el IVA, que son las tasas sobre los rendimientos del trabajo y el consumo, los estados tienen que aumentar su deuda, a menos que quieran rebajar radicalmente los servicios que prestan. Su deuda tiene que ser financiada por los bancos, o mediante la inflación, que podría agravar el problema, al perder competitividad con las mercancías de los países emergentes como China y la India, con lo cual se cae en el dilema que ha estudiado Thomas Piketty (2014). Un dilema del que sólo se podría salir haciendo que las rentas del capital pasen a tributar muchos más. Sin embargo, como el mercado del capital es global eso sólo se podría lograr con acuerdos internacionales, muy difíciles porque la economía interfiere con el poder militar, cuando se mueve a escala global.

La desindustrialización de los EE.UU. no ha afectado al campo militar. El gasto militar de los EE.UU. ha sido en el año 2016 de 604.452 millones de dólares, frente a los 145.039 de la China. Su proporción en el PIB es del 3.26\%, mientras que en la China es del 1,27\%. Uno de cada diez empleos en los EE.UU. está en las fuerzas armadas o en las empresas relacionadas con ellas en su mantenimiento y en la producción y diseño y creación en nuevas armas (Bourke, 2014). El Pentágono contribuye con importantes fondos para la investigación en las universidades de la Ivy League, y lo mismo ocurre en Inglaterra con universidades como Oxford y Cambridge. Todo el conglomerado militar industrial mueve la mayor parte de la inversión en investigación en todo el mundo, sólo seguida por las industrias farmacéuticas, gigantescas proveedoras de los sistemas de sanidad públicos. Se defiende la necesidad constante de crear nuevos sistemas de armamento que ni siquiera los militares demandan, porque la innovación propia es rápidamente equilibrada por la del enemigo y se crea una carrera de armamentos absurda. Se ha desarrollado una ideología militar, llamado el "orientalismo militar" (Porter, 2013) que desprecia las formas de hacer la guerra no basadas en las tecnologías más complejas, cuyo valor en los casos de Vietnam, Irak o Afganistán ha quedado en entredicho. Las universidades de élite no solo son financiadas parcialmente por el Pentágono y colaboran con las industrias de defensa, sino que favorecen y hacen propaganda del reclutamiento en las fuerzas armadas, cuyos valores comparte y de cuyas inversiones se benefician. El capitalismo del siglo XXI es pues global, financiero y va unido a los intereses militares y estratégicos globales. $Y$ lo hace sin complejos y con claridad, teniendo muy claro que las universidades, como el resto de los sistemas educativos, constituyen otra ficha más de su ajedrez. 
¿Qué pasa con nuestras universidades? ¿Están en el capitalismo global? Rotundamente no. ¿Cuál es su situación? Para intentar comprenderla analizaremos tres niveles. En primer lugar, el nivel ideológico, que es el que consolida las representaciones fetichistas en los campos económico e institucional; y en segundo lugar el nivel psicosocial, que es aquel en que sus miembros asumen su propia alienación. Como las representaciones ideológicas se caracterizan por tener un carácter anónimo, que es imprescindible para que puedan ser confundidas con la realidad, lo que sería imposible si fuesen consideradas creaciones de personas concretas, iremos estudiado paso a paso las tesis ideológicas tal y como se le presentan a la colectividad y son asumidas por ella como parte de la realidad.

Tesis principal:

Las universidades se definen básicamente por el papel central que desempeñan en la economía de cada país. La importancia de las universidades puede medirse por el índice porcentual que les corresponde en el PIB, y cuanto más elevado sea ese porcentaje mayor desarrollo económico y social tendrá un determinado país. Y ello es así porque en el momento presente el desarrollo económico depende básicamente de la capacidad de innovación tecnológica de la industria de cada país. Como los mercados son enormemente competitivos la única manera de lograr un beneficio más elevado es creando una ventaja competitiva nueva. Es decir, creando nuevos productos de base técnica que capten determinados nichos de mercado o que creen nichos nuevos. $\mathrm{Y}$, dado que la innovación industrial no es posible sin procesos de investigación que hagan posible el diseño y la producción rentable de nuevas mercancías, deberemos considerar la inversión en investigación como un componente esencial de la economía española. Nuestras universidades son capaces de afrontar este reto porque han sido modernizadas y poseen una estructura y organización racionales. Forman un sistema regulado legalmente y que está controlado con rigurosos criterios de calidad en el funcionamiento de todos y cada uno de sus aspectos. $Y$ además han creado un capital humano altamente cualificado equiparable al de los países más desarrollados. Y sólo tienen un defecto que lastra su desarrollo y les impide cumplir su papel económico y social: su escasa financiación. Y, por último, dado que la inmensa mayoría de nuestras universidades son públicas, lo que es plenamente cierto en el campo de la investigación, consecuentemente la solución de sus problemas y del problema esencial de la economía española es incrementar el gasto público, para mejorar la competitividad de la economía en general. 
Comenzaremos por dar algunas cifras, tomadas del estudio dirigido por Antoni Castells, Manuel Castells, Josep Oliver, Emilio Ontiveros y Martí Parellada (2013). La universidad española en el año 2013 atendía a un millón y medio de estudiantes de primero y segundo ciclo y de grado y a más de 113.000 estudiantes de máster. En ese año se habrían graduado 222.000 estudiantes y 50.000 habrían conseguido el título de máster. Todo ello con unas plantillas de 115.000 profesores y de unos 60.000 empleados que ejercían las funciones administrativas y de servicios. A la universidad le correspondió el $29 \%$ del gasto en I+D de la economía española y en ella trabajan el $48 \%$ de los investigadores, desarrollando el $17 \%$ de las patentes, pero copando el $69 \%$ de las publicaciones científicas. El gasto interno en I+D es muy bajo si se compara porcentualmente en el PIB con otros países, como Israel, que tiene el gasto más elevado porcentualmente o Finlandia. En el 2011, antes de la crisis ese porcentaje había sido del 1,3\%., mientras que en Alemania, por ejemplo sería del 3\%., pero debemos señalar que una cosa es la inversión en I+D en el marco empresarial e industrial, que es la que puede crear riqueza, ya que entra dentro de los procesos productivos, y otra cosa muy distinta es el gasto público en I+D, que puede no crear ninguna riqueza si la investigación se mide básicamente por la cantidad de publicaciones en las revistas científicas internacionales. Esas revistas se editan en régimen prácticamente de monopolio por dos o tres grandes grupos editoriales, tienen unos elevadísimos costes de suscripción, no remuneran a los autores que publican en ellas, que a veces incluso tienen que pagar por publicar en las mismas, y hacen públicos los resultados para que cualquier empresa pueda utilizarlos en sus procesos productivos.

Desde el año 2001 al 2011el número de papers publicado por españoles pasó de unos 20.000 a unos 80.000 , incrementándose mucho la ratio en relación con el total de la publicación en resto del mundo. Sin embargo, en ese mismo período el incremento porcentual de las patentes registradas por las universidades sólo pasó del $8 \%$ del total al $16 \%$. El otro $84 \%$ de las patentes registradas correspondió a la empresa privada. La financiación del I+D en Japón corresponde en un $70 \%$ a la empresa privada y un $30 \%$ al sector público. Lo mismo ocurre en Corea del Sur o China, mientras que en los EE.UU. o Alemania la ratio es del 60\% y 40\%. En España, por el contrario, el $30 \%$ corresponde al sector privado y el $70 \%$ al público o a la financiación con capital extranjero. La economía española es en cierto modo la imagen invertida de la economía alemana. En Alemania de cada 3 jóvenes entre los 18 y 21 años, 2 están en la FP y uno en la universidad, en España la ratio es la contraria. Pero 
curiosamente en Alemania las ocupaciones que requieren alta cualificación son el 38,9 $\%$ de las totales, mientras que en España son sólo el 29 \%, 9,9 puntos porcentuales por debajo. De la misma manera en Alemania el número de titulados superiores es el $28,1 \%$ de la población del país, mientras que en España es el 32,3 \%. Lo que es sorprendente si tenemos en cuenta la diferencia de población, de riqueza y del nivel de industrialización que existe entre ambos países. Está claro que en España sobran titulados superiores, porque en el $35 \%$ de los titulados realizan trabajos que no requieren ninguna cualificación superior, mientras que en Alemania sólo lo hacen el $12 \%$. Si comparamos los niveles de renta salarial entre los 25 y 64 años, veremos que en España la diferencia de sueldo entre titulados superiores que ejercen un trabajo específico de su titulación y el nivel medio de salario es del $155 \%$, mientras que en Alemania es del $180 \%$ y en los EE.UU. del $185 \%$. De lo que se puede deducir la escasa rentabilidad laboral que tienen en España los estudios superiores. Se puede concluir, además, en palabras de estos autores que: "más allá del debate sobre si el sistema universitario español tiene un número demasiado elevado de universidades, el debate debería recaer en como reorganizar el mapa de titulaciones" (Ibídem, p. 6). Porque no es sólo que las cifras globales hablen por sí mismas, sino que se ofrecen multiplicidad de titulaciones en la misma comunidad autónoma. Todas las provincias andaluzas tienen, por ejemplo, una facultad de Historia. La universidad de Vigo tiene dos facultades de derecho a menos de $100 \mathrm{~km}$. conectados por una autopista. Y es que además de no existir coordinación entre las 17 comunidades autónomas que conforman el Estado, ni siquiera dentro de cada una de ellas las universidades se coordinan entre sí. Las universidades españolas no forman un sistema, sino que son un conglomerado explicable por las circunstancias históricas de su nacimiento, y porque en ellas lo nuevo se suma a lo existente, pero no se integra en él. Por razones demográficas y de otro tipo el número de alumnos había descendido entre el 2004 y el 2009, recuperándose en el 2011 en el nivel del 2007, pero no alcanzando ya el nivel anterior al 2004. Sin embargo, el número de profesores ha continuado creciendo, y las reducciones de plantilla se debieron básicamente a las jubilaciones.

Si analizamos las fuentes de su financiación veremos que la financiación es básicamente pública, que sus ingresos por investigación son muy bajos, ya que los proyectos que pueden conseguir de la financiación pública sus profesores son financiados de modo finalista, de tal modo que el dinero conseguido se gasta en la realización del propio proyecto, sin que apenas repercuta en los ingresos de cada universidad, a pesar de que retienen un pequeño porcentaje a cambio de los servicios 
que ofrece a los investigadores y sus equipos. En el campo de la investigación universitaria se está produciendo además una división del trabajo en dos grupos antagónicos. El de los profesores funcionarios que tienen garantizados sus ingresos y que pueden mejorarlos con la investigación, y el de los jóvenes contratados y precarios, cuyos sueldos son normalmente bajos, a pesar de que se les exige un nivel de preparación y de rendimiento muy elevados, y a los que es imposible garantizarles un futuro laboral estable, pues solo pueden ser profesores en universidades que tienen unas plantillas muy excedentarias. Se da además el caso de que la bajada de los salarios, que es un fenómeno global en la economía convertida en finanzas, tal y como había señalado Piketty, también está afectando de un modo drástico a profesores e investigadores en los EE.UU. y los países más desarrollados (Donoghue, 2008; Newfiled, 2003). Los salarios se han reducido a la mitad o la tercera parte, en muchos casos, entre otras cosas por la competencia que la oferta de trabajo cualificado con salarios a la baja por parte de los científicos e investigadores de la China o la India está produciendo en los propios EE.UU. y Europa. Buena prueba de ello es la incapacidad de devolución de los créditos bancarios para el estudio en las universidades de élite que ha generado una burbuja universitaria de unos 1.200.000 millones de dólares y que está a punto de estallar, si no se frena a tiempo. Deberíamos preguntarnos si los apóstoles españoles de la ideología del mercado y la innovación están dentro o fuera de la realidad. Sin duda cumplen muchas de las características que acompañaron el nacimiento del capitalismo, aunque con matices. Veámoslo.

Se mueven en un mundo global, en el que son capaces de buscar y analizar información y crear nueva información y conocimiento, ya que las ciencias, o son globales o no son ciencias. Pero en contraste con ello defienden un sistema universitario aislado, con sus universidades divididas entre 17 Autonomías no coordinadas, cerradas cada una en sí misma también dentro de la propia Autonomía y con unos cuadros de profesores que no pueden trasladarse de una universidad a otra siendo ya funcionarios. A lo que se añade la tendencia al reclutamiento endogámico como forma de defensa frente a la competencia y como estrategia de supervivencia, ya que en la lucha por la vida es necesario utilizar las mismas armas que el adversario o mejores, si no queremos ser aniquilados. $Y$ por eso yo no puedo ser generoso si todos tienden al egoísmo y la endogamia. Hay un mundo global de la información, pero esa información se queda aislada en una cápsula porque solo sirve para publicar artículos de alto coste de producción en redes cerradas y hacerlo además, no solo de modo gratuito, sino comprando su uso con la suscripción. La 
producción de innovación científica no genera riqueza, porque no se produce ni se vende nada, sino que sólo se gasta, porque para estar al día es necesario renovar equipamientos de todo tipo y estar dispuesto a consumir la información que se ofrece a la venta en el mercado editorial y otros mercados científicos. Si el capitalismo exigió romper las trabas sociales, legales, geográficas y mentales que permitiesen el establecimiento del mercado y su búsqueda del equilibro, en las universidades españolas ocurre todo lo contrario, porque son organismos rígidos y carecen de capacidad de adaptación y de renovación institucional. $Y$ además porque la mentalidad de sus profesores tiende a ser dogmática, al estar envuelta en una ideología que favorece la reificación de las relaciones académicas, o lo que es lo mismo, que tiende a imponer la idea de que el orden académico, que es convencional y cambiable, es natural, inmutable e inapelable.

Pero la cuestión se agrava aún más si tenemos en cuenta que la economía global se ha convertido en financiera, que es una economía monetaria y de juegos monetarios y en la cual los beneficios económicos dependen cada vez más de la especulación y cada vez menos de la producción y la innovación. Se puede ganar más en las bolsas que en el mercado real, pero es que además en el campo de la innovación los beneficios de una patente corresponden más a quien la compra y puede luego revenderla para su uso industrial, que a quienes han trabajado largos años y han invertido dinero en su creación. También la especulación se está comiendo a la innovación. En todos los casos menos uno, el de la investigación militar, que no se hace en primer lugar para el mercado, sino para la defensa, y cuyos resultados nunca se publican. Durante la Segunda Guerra mundial, por ejemplo, norteamericanos y alemanes observaron que ambas potencias estaban intentando conseguir la bomba atómica porque habían desaparecido de las revistas nuevas todas las publicaciones relacionadas con la posible producción de la bomba (Hughes, 2003; Ball, 2013). Sin embargo, esa investigación no ocupa un papel importante en las universidades públicas españolas, que más bien tienden a pronunciarse solemnemente en contra del I+D militar, con lo que su aislamiento de las redes de la investigación se hace aún mayor.

En la ideología académica española el PIB ha alcanzado el nivel de un ser mitológico. Se habla del porcentaje del PIB en I+D sin distinguir la inversión pública de la privada. $Y$ como se utiliza la palabra ciencia en general no se distingue aquel conocimiento que puede formar parte de un proceso productivo del que nunca entrará en él. Además, no se conocen los cuatro componentes del PIB y se confunden gasto 
con inversión, cuando pueden ser todo lo contrario. Todo gasto público se hace a costa de detraer del mercado mediante los impuestos una parte de la riqueza de los trabajadores, sobre todo, y de las empresas. Es cierto que con el gasto público se puede invertir en empresas a veces, pero esa inversión de capital no tiene por qué ser para investigación. Se puede ayudar a que una empresa exista con exenciones fiscales, facilitando la contratación o invirtiendo en procesos ya consolidados que no necesiten innovación. En España más del 90\% de las empresas son pequeñas o medianas y el número de trabajadores autónomos es muy elevado. Es evidente que ese tipo de empresas no pueden soportar un gasto en investigación. Pero es que además en el PIB una parte esencial es el turismo, que tampoco la demanda, la agricultura, que supera el $11 \%$ del PIB. Y la exportación industrial se centra en el campo de la automoción en el que todas las empresas instaladas en España son extranjeras, por lo que sus departamentos de I+D suelen estar en sus países de origen. Esta es la realidad económica de un país con una deuda pública que supera a su PIB, que ha venido arrastrando un considerable déficit público, y que tiene considerables problemas sociales con sus sistemas de pensiones, su sanidad y su mercado laboral de baja cualificación y en el que los titulados superiores muchas veces no pueden encontrar un trabajo adecuado.

Este es el mundo, pero el mundo de la ideología es el mundo al revés, como la imagen de la cámara oscura. En la ideología académica se ha creado el fetichismo del conocimiento. En él la ciencia es una variable independiente de la que dependen todas las demás. Si Arquímedes pidió una palanca para mover el mundo, nuestros académicos y políticos piden más dinero para transformar el mundo sólo con el conocimiento y la economía del conocimiento, que en su caso trae consigo el desconocimiento real de la economía. ¿Por qué piensan así? Pues porque se han creado un mundo propio, que no tiene nada que ver ni con el mundo real, ni tampoco con el mundo del conocimiento y la ciencia reales. Es el mundo de la calidad y el control administrativo. Un mundo que es posible en las universidades públicas porque no dependen del mercado en sus ingresos y porque se controlan y gobiernan a sí mismas, teniendo como objetivo básico la defensa de los intereses profesionales de sus miembros, sobre todo de sus profesores. Si hay un gasto que ha crecido sin parar en las universidades es el gasto administrativo. La universidad de Santiago, por ejemplo, tiene en el 2018, unos 2000 profesores para 25.000 alumnos y unos 1300 administrativos y personal de servicios. Los procedimientos administrativos se han hecho cada vez más complejos y largos, a la vez que ineficaces, por lo que requieren 
miles y miles de horas de trabajo administrativo de los profesores, además del de los funcionarios correspondientes. Todo este trabajo no se hace para cambiar las cosas tras diagnosticar su situación, como hace el capitalista en el mercado, sino que forma parte de un ritual de control y sumisión del personal docente y del alumnado. No ha habido ninguna reforma global de la universidad desde la LRU de José María Maravall, que entró en vigor hace unos 32 años. Nadie quiere ni reformar la universidad ni adaptarla a sus nuevos contextos históricos ni coordinar nada ni hacer a las universidades realmente más eficientes y rentables, porque ello supondría perder cuotas de poder y control. Ni las Autonomías están dispuestas a ceder nada, ni los rectores de cada una de sus universidades tampoco. Podríamos entonces estar ante una especie de sociedad estamental bloqueada y cerrando filas en torno a sus privilegios. $Y$ ante eso estamos, pero la imagen que se da en la ideología académica es la contraria.

Según el fetichismo del control racional todo debe ser estudiado, pautado, planificado y evaluado sin cesar. Para ello se crean palabras vacías, como competencias y habilidades, o calidad, que nunca se definen ni se quieren definir porque se trata de utilizarlas como lemas e instrumentos de control. Esos lemas tienen que ser sencillos para hacer posible que personas cada vez menos inteligentes y carentes de sentido crítico, pero con ambición de poder y de promoción, formen la columna vertebral y la élite del sistema. De un sistema en el que no importa nunca ni la realidad ni el contenido, sino solo el procedimiento. Como podremos ver con algunos ejemplos.

Es evidente que para valorar el trabajo científico hay que tener conocimientos científicos. Sólo los especialistas en un campo saben lo que de verdad es innovador en él. Pero eso supone reconocer que la realidad es múltiple y entonces no se puede controlar, y por eso se ha creado un primer sistema de control del conocimiento con las evaluaciones científicas universales. Sus principios son los siguientes: a) el conocimiento o se mide o no es conocimiento; b) como no hay átomos de conocimiento en ningún campo ni en muchos campos transversales es necesario crear la unidad de medida; c) esa unidad de medida es la publicación, básicamente en forma de artículo; d) como hay que valorar de modo general no se puede leer ni analizar y por eso es necesario sustituir los criterios de valor internos por los criterios de valor externos; e) lo que da valor es el lugar, la revista o editorial en dónde algo se publica; f) ese lugar en el mercado tiene el valor de la marca comercial; g) es la marca la que da valor y por eso es necesario ampararse bajo el poder de la marca; $h$ ) pero 
esas marcas tiene unos criterios de selección de temas, formas y protocolos de admisión, por lo cual hay que someterse a ellos o morir (Day, 2007). Por eso ya son las propias empresas editoriales las que enseñan cómo hay que publicar. Todos estos criterios se utilizan de modo orientativo en los campos científicos en todo el mundo, aunque no en los de las humanidades y las ciencias jurídicas. Pero la contratación de profesores e investigadores en la industria y las universidades de los principales países la hacen expertos o autoridades que saben a quién quieren contratar y cómo hacerlo. Por eso no necesitan los cientos de tablas y baremos que se han creado en España. No sólo los considerarían falsos, sino también ridículos, porque las industrias y las universidades sobreviven o desaparecen en un mercado y un mundo reales, según funcionen bien o mal y no según los parámetros que dicten los burócratas. $\mathrm{Al}$ contrario que en España en dónde las universidades públicas están blindadas ante la realidad. El sistema de control de las publicaciones es la base del control de las personas, porque sin publicaciones no se puede acceder a la carrera docente o investigadora Para hacerlo, siguiendo los consejos de Abby Day en su libro citado, se debe hacer lo siguiente: no quieras ser original, publica lo que te piden y en la forma que te lo pidan y así acumularás un patrimonio o capital académico, que será el que te otorgue tu identidad profesional.

El control de las publicaciones en España se hace por parte de agencias, como la ANECA, cuyo coste anual supera los 3 millones de euros y que tiene réplicas clónicas en todas las comunidades autónomas. Ahí se fijan los criterios, se establecen los protocolos de control y evaluación y son ellas las que nombran a los evaluadores dispuestos a someterse a sus criterios y a hacer un gigantesco trabajo, científicamente inútil, pero académicamente esencial. Los criterios españoles, nacionales o autonómicos solo sirven en nuestro país. Sobre todo en el campo de las humanidades en el que la calificación de las revistas y editoriales de lengua española tienen criterios específicos, dándose el caso de que lo que aquí se considera como de primera calidad en el resto del mundo puede ser de calidad baja o nula. Los profesores y estudiantes comienzan por someterse al sistema de control de publicaciones, pero eso no es suficiente para dominarlos, pues tienen que admitir los criterios de elaboración de los planes de estudio y los controles de su docencia basados en otras normas de validez universal, elaboradas por burócratas y pedagogos que han generalizado los parámetros de la enseñanza media en todos los niveles de la educación superior. Como esos criterios también son sencillos y vacíos, así se ha conseguido que miles de funcionarios y profesores puedan pasar a controlar todos los planes de estudio y 
su implementación en su papel de evaluadores de la calidad docente de todos los demás. Pero es que además se ha introducido un nuevo factor en el proceso. El dinero público cada vez más se concentra en la financiación de proyectos y grupos de investigación. Esos grupos técnicamente sólo son consumidores de renta pública. El Estado ofrece dinero y ellos lo captan, ajustándose a los criterios de su oferta. Pero ese dinero es escaso y por eso todos compiten por él como consumidores acaparadores de renta. $Y$ tiene que ser acaparadores porque ese dinero es solo gasto y no inversión: gasto en personal, instrumentos, reactivos, estancias y dietas, publicaciones, etc. Conseguir dinero es conseguir prestigio, porque como la investigación no se puede medir por el contenido es necesario crea otro parámetro externo de medida: el gasto. Lo que se hace de un modo perverso, pues no es mejor quién obtiene mejores resultados con menos dinero, sino quién más gasta, sin más. Quién más ha gastado es mejor, porque se supone que el dinero solo se concede en función del mérito que consiste en conseguirlo de manos de funcionarios y profesores burócratas. Se produce así lo que en las universidades norteamericanas se llama el "efecto san Mateo", siguiendo el pasaje evangélico que dice: "en verdad os digo que al que tiene se le dará y al que no tiene incluso eso se le quitará". Este gasto es lo que se llama consumo conspicuo, siguiendo la terminología de Throsten Veblen, es decir, una forma de exhibir el prestigio, porque el prestigio que no se pueda exhibir no puede existir.

A eso debemos añadir que el acceso a puestos docentes o de investigador es lo que cierra el círculo académico, ya que es lo único que puede dar seguridad laboral y que permite seguir acumulando ese prestigio que solo es reconocido dentro de la academia. Nadie lo reconoce en el mundo exterior, porque el sentido común se impone y todo el mundo sabe que un especialista lo es por lo que sabe, que el valor de las cosas depende de su efectividad y no de parámetros y de rankings de citas, que se perciben como una expresión de un narcisismo infantil. Esto es así porque nada real depende de esos rankings, ya que las universidades ni se crean ni se destruyen en función de ellos, solo se transforman a veces, para no cambiar en lo esencial.

Todos los grupos y corporaciones tienen sistemas de valores propios, que a veces no son comprensibles desde fuera de la comunidad. Así ocurre en los ejércitos, la policía, la sanidad, las iglesias y las empresas. Y el mundo académico no iba a ser menos. Los valores académicos derivan en parte de los valores religiosos, porque las universidades eran esencialmente instituciones eclesiásticas hasta el siglo XIX 
(Collins, 2005). Los miembros de las universidades se reconocen entre sí mediante el sistema de valores que comparten, pero también necesitan de reconocimiento del mundo exterior. Ese reconocimiento puede deberse a que se les reconozca su papel esencial en la formación religiosa, filosófica, o sea en la creación de los sistemas sociales y políticos de valores, o en su papel como formadores de profesionales, como en otro tiempo fueron los clérigos, y siguen siendo los profesionales del mundo del derecho, la educación, la salud y el conocimiento científico y técnico. Pero todos esos profesionales tienen que demostrar su valor fuera del mundo académico. En España eso ocurre cada vez menos porque sobran titulados superiores, y esos titulados caen en el subempleo o pasan directamente al ejército laboral no cualificado por necesidad. La universidad o sirve al mundo real o no sirve para nada, y esto es lo que está ocurriendo cada vez más. Eso es así, pero no es percibido así por muchos de sus miembros porque están alienados por el fetichismo académico. Su alienación consiste en ser incapaces de percibir lo que uno es en el mundo real y sobre todo en el mundo de la producción. Un profesor debería saber que es un profesor, cuál es su función, cuales su trabajo, para qué sirve, y sobre qué valores se asienta su disciplina. $Y$ también debería saber que ser profesor en una universidad pública supone un compromiso con los valores de la universidad y de la función pública. $Y$ en ese compromiso a veces es necesario defender esos valores frente a los intereses corporativos o las presiones políticas y económicas, porque esa es una de las funciones esenciales del Estado y las universidades públicas, pues forman parte de él. Esto sin embargo está dejando de ser así, debido a la alienación del profesorado y el alumnado, que se corresponde con una situación política global en las que han desaparecido las ideologías políticas y con ellas la esperanza de cambiar el orden del mundo en la política, la economía o el dominio militar.

La política española es un juego verbal, basado en lemas, programado con imágenes e integrado en el mundo de la comunicación digital, en el cual los textos mínimos de los tuits y los lemas machacados hasta la saciedad han conseguido anular la capacidad de pensar y analizar la realidad social. Pero junto a esto existe el mundo de la administración, que controla un tercio del PIB con el gasto público y está administrado por economistas, juristas y expertos de todo tipo, que pueden trabajar por igual para cualquier partido sin exclusión. Son esos expertos del estado endeudado y sostenido por la banca, los aliados naturales de banqueros y empresarios y el modelo que los profesores alienados quieren imitar. Por eso se pasan tan fácilmente a la política, y a la empresa, aunque esto es más difícil porque ahí no 
los suelen querer. En el mundo alienado de la universidad nadie sabe lo que en realidad es. El profesor cree ser una palanca esencial de la riqueza del país, cuando ni es ni será verdaderamente rico. Únicamente podrá captar un poco más del dinero público, pero lo hará a costa de su servidumbre y de aquellos que ya son sus siervos académicos, esperando también algún día llegar a ser pequeños señores. Sus lemas serán siempre palabras abstractas y vacías: ciencia, inversión, I+D, riqueza, calidad, competencia, habilidad, porque esas palabras son las que le explican el papel que está representando sin saberlo, en tanto que está alienado. Permiten además enmascarar la realidad y hacer que la apariencia y la esencia nunca coincidan. Alienados, hechizados por la ideología de la economía competitiva y de la economía del conocimiento, los profesores, alumnos y académicos en general, cada vez más, compran con su sumisión institucional, política e ideológica los pequeños privilegios que les permiten vivir en el patio de los mandarines (Ringer, 1969³), como lo hicieron los grandes profesores alemanes, valorados por sus grandes conocimientos, que no por unos burócratas. Quizás por eso sería bueno concluir este ensayo con un verso de Gootfried Keller:

El tiempo es blanco pergamino

y el hombre escribe, en cada hoja

con tinta que es su sangre roja

hasta que lo hunde el torbellino.

\section{REFERENCIAS}

Anderson, E. (1993). Value in Ethics and Economics. Harvard: Harvard University Press.

Ball, Ph. (2013). Serving the Reich. The Struggle for the Soul of Physic under Hitler. London: The Bodley Head.

Barker, R. W. (2005). Capitalism's Achilles Heel. Dirty Money and How to Renew the Free -Market System. New Yersey: John Wiley.

Beckert, S. (2015). Empire of Cotton. A New History of Global Capitalism. Hardmondsworth: Penguin.

\footnotetext{
${ }^{3}$ Fue este autor quién utilizó el concepto de mandarín para estudiar al profesorado alemán. A unos profesores, altamente reconocidos que siempre quisieron vivir al margen de la política y el mundo real, y lo consiguieron, hasta que con la llegada del nazismo tuvieron que someterse a él.
} 
Bermejo Barrera, J. C. (2008). La Aurora de los enanos. Decadencia y caída de las universidades europeas. Madrid: Foca.

Bermejo Barrera, J. C. (2009). La fábrica de la ignorancia. La universidad del como si. Madrid: Akal.

Bermejo Barrera, J. C. (2010). The Limits of Knowledge and the Limits of Science. Santiago de Compostela: Universidad de Santiago de Compostela.

Bermejo Barrera, J. C. (2011). La maquinación y el privilegio. El gobierno de las universidades. Madrid: Akal.

Bermejo Barrera, J. C. (2012). La consagración de la mentira, entre la realidad y el silencio. Madrid: Siglo XXI.

Bermejo Barrera, J. C. (2015). La tentación del rey Midas. Para una economía política del conocimiento. Madrid: Siglo XXI.

Bermejo Barrera, J. C. (2017). Rectores y privilegiados. Crónica de una universidad. Madrid: Foca.

Bourke, J. (2014). Wounding the World. How Military Violence and War-Play invade our Lives. Londres: Virago.

Castells A., Castells, M., Oliver, J., Ontiveros, E. y Parellada, M. (2013). La reforma de la universidad. Un impulso a la competitivad de la economía española, Policy Brief, 4, 1-18.

Collins, R. (2005). Sociología de las filosofías. Una teoría global del cambio intelectual. Barcelona: Hacer.

Day, A. (2007). How to get Research published in Journals. Hampshire: Gower.

Donoghue, F. (2008). The Last Professors. The Corporate University and the Fate of the Humanities. Nueva York: Fordham University Prees.

Dunbar, N (2011). The Devil's Derivatives. Boston: Harvard Bussiness Review Press.

Friedman, M. y Schwart, J.A. (1971). A Monetary History of the United States, 18671960. Princeton: Princeton University Press.

Georgescu-Roetgen, N. (1971). The Entropy Law and Economic Process. Harvard: Harvard University Press.

Grazia, V. de. (2005). Irresistible Empire. America's Advance Througth 20th Century Europe, Harvard-Cambridge: Belnap Press.

Groeben, D. (2012). En deuda. Una historia alternativa de la economía. Barcelona: Ariel.

Hughes, J. (2003). The Manhattan Proyect. Big Science and the Atom Bomb, Londres: Revolutions in Science. 
Kain, Th. (1988). Marx on Ethics. Oxford: Clarendon Press.

Kofman, S. (1975). Cámara oscura. De la ideología. Madrid: Taller ed.

Leopold, D. (2012). El joven Karl Marx. Filosofía alemana, política moderna y realización humana. Madrid: Akal.

Levitt, S. D y Dunbar, S. J. (2005). Freakinomics, Barcelona: Círculo de Lectores.

Lindert, P. (2011). El Ascenso del sector público, I y II. México: Fondo de Cultura Económica.

Madison, A. (2007). Contours of the World Economy, I- 2030 AD. Oxford: Oxford University Press.

Marx, K. (2017). El Capital. Crítica de la economía política. Madrid: Siglo XXI.

Marx, K. y Engels, F. (2014). La ideología alemana. Madrid: Akal.

Miller, P. (2015). Peiresc's Mediterranean World. Harvard: Harvard University Press.

Naredo, J. M (2003). La economía en evolución. Historia y perspectivas de las categorías básicas del pensamiento económico. Madrid: Siglo XXI.

Newfiled, Ch. (2003). Ivy and Industry. Bussiness and the Making of the American University, 1880-1980. Durham: Duke University Press.

Passet, R. (2010). Les grandes Répresentations du monde et de l'économie a travers I'Histoire, París: Les Linsqui Libérent.

Piketty, Th. (2014). El Capital en el siglo XXI. México: Fondo de Cultura Económica.

Plamenatz, J. (1983). La ideología. México: Fondo de Cultura Económica.

Porter, P. (2013). Military Orientalism. Eastern War Through Western Eyes. Oxford: Oxford University Press.

Ringer, F. (1969). The Decline of the German Mandarins. The German AcademicCommunity, 1890-1933. Londres: Wesleyan University Press.

Schumpeter, J. R. (1954). History of EconomicAnalysis. Oxford: Oxford University Press.

Stein, J. (2010). Pivotal Decade. How the United States traded Factories for Finance in the Seventies. New Haven: Yale University Press.

Taibi, M. (2011). Cleptopía, Madrid: Lengua de Trapo, Madrid.

Werner, S. (1967). II Capitalismo moderno, t. I, II, III, Torino: UTEH. 